\title{
SOLUTION OF ADHESIVE CONTACT PROBLEM ON THE BASIS OF THE KNOWN SOLUTION FOR NON-ADHESIVE ONE
}

\author{
UDC 539.6
}

\author{
Valentin L. Popov \\ Berlin University of Technology, Berlin, Germany
}

\begin{abstract}
The well-known procedure of reducing an adhesive contact problem to the corresponding non-adhesive one is generalized in this short communication to contacts with an arbitrary contact shape and arbitrary material properties (e.g. non homogeneous or gradient media). The only additional assumption is that the sequence of contact configurations in an adhesive contact should be exactly the same as that of contact configurations in a non-adhesive one. This assumption restricts the applicability of the present method. Nonetheless, the method can be applied to many classes of contact problems exactly and also be used for approximate analyses.
\end{abstract}

Key Words: Adhesion, Normal Contact, Heterogeneous Media, Effective Surface Energy

\section{INTRODUCTION}

The present short communication is publication of a short memo written on May, $14^{\text {th }}$, 2015, and not published at that time as the field of applications of the obtained results seemed to be very narrow. It was communicated privately to colleagues and published for a restricted set of adhesive contact problems in [1]. Since then, it has been applied to a variety of problems including axially symmetric contact ones without a compact contact area [2] just as it has been systematically applied to a large variety of contact problems in the recent Handbook on Contact Mechanics [3]. However, the derivation and results are more general than the cases considered in [2] and [3]. They are based solely on existence of some force-indentation and area-indentation dependencies for non-adhesive contacts. In the present paper we provide a general derivation which is even applicable to the situations where the surface energy is a function of the coordinates.

Received January 05, 2018 / Accepted January 31, 2018

Corresponding author: Valentin L. Popov

Technische Universität Berlin, Sekr. C8-4, Straße des 17. Juni 135, D-10623 Berlin

E-mail: v.popov@tu-berlin.de 


\section{AXIALly SyMmetric CONTACT PROBLEM}

Consider indentation of a profile $z=f(r)$ into an elastic medium with plane surface. The medium is assumed to be homogeneous in-plane, but may be heterogeneous in the $z$ direction (e.g. a layer or a gradient medium and so on). We assume that indentation depth $d$ is much smaller than the characteristic size of heterogeneity, while this may be not the case for contact radius $a$. We assume that the non-adhesive normal contact problem for this shape and this medium has been solved, so that the dependences of normal force $F_{N}$ and of the contact area on the indentation depth are known. Each of these three quantities determines uniquely two others, so that we can consider normal force $F_{N, \text { n.a. }}(a)$ and indentation depth $d_{n . a}(a)$ as known functions of the contact radius, too. We can further define the potential energy of non-adhesive contact, $U_{\text {n.a. }}(a)$ and the contact stiffness

$$
k_{n . a .}(a)=\frac{\mathrm{d} F_{N, n . a}(a(d))}{\mathrm{d} d} .
$$

which also can be considered as a known function of the contact radius.

Now let us consider an adhesive contact under assumptions of the JKR-theory (range of interaction of adhesive forces much smaller than any characteristic size of the problem, so to say zero) and characterize adhesion with the work of detachment of surfaces per unit area, $\Delta \gamma$.

The solution to this problem is given by the following set of equations:

$$
\begin{gathered}
d=d_{n \cdot a .}(a)-\Delta l_{c}(a), \\
F_{N}(a)=F_{N, n . a .}(a)-k_{n . a .}(a) \cdot \Delta l_{c}(a)
\end{gathered}
$$

with $\Delta l_{c}(a)$ given by the following equation:

$$
\Delta l_{c}(a)=\sqrt{\frac{4 \pi a \Delta \gamma}{\mathrm{d} k_{\text {n.a. }}(a) / \mathrm{d} a}} .
$$

Eqs. (2) and (3) give in implicit form the dependence of the normal force (in the adhesive contact) on the indentation depth thus solving the adhesion problem.

Let now prove the Eq. (4). If we indent the profile up to contact radius $a$, then the potential energy in this state will be $U_{\text {n.a. }}(a)$ and indentation depth $d_{\text {n.a. }}(a)$. The force in this moment will be $F_{N, n . a}(a)$. Now let lift the indenter by $\Delta l$ without changing the contact area. During this process the stiffness of the contact remains constant and equal to $k_{\text {n.a. }}(a)$. Therefore, the force will change according to

$$
F_{N}(a)=F_{n . a .}-k_{n . a .}(a) \Delta l
$$

and the potential energy will be equal to

$$
U(a)=U_{n . a .}(a)-F_{N, n . a .}(a) \Delta l+k_{n . a .}(a) \frac{\Delta l^{2}}{2} .
$$


The new indentation depth will be

$$
d=d_{\text {n.a. }}(a)-\Delta l
$$

Let us now solve (7) with respect to $\Delta l$ und insert it into Eq. (6):

$$
U(a)=U_{n . a .}(a)-F_{N, n . a .}(a)\left(d_{n . a .}(a)-d\right)+k_{n \cdot a .}(a) \frac{\left(d_{n . a .}(a)-d\right)^{2}}{2} .
$$

The total energy (with consideration of the adhesion energy is equal to

$$
U_{t o t}(a)=U_{n . a .}(a)-F_{N, n . a .}(a)\left(d_{n . a .}(a)-d\right)+k_{n . a .}(a) \frac{\left(d_{n . a .}(a)-d\right)^{2}}{2}-\pi a^{2} \Delta \gamma .
$$

Equilibrium value of $a$ corresponds to the minimum of this energy with respect to $a$ for a constant indentation depth $d$. To determine the minimum, we let the derivative be zero:

$$
\begin{aligned}
& \frac{\mathrm{d} U_{t o t}(a)}{\partial a} \\
& =\frac{\mathrm{d} U_{n . a .}(a)}{\mathrm{d} a}-\frac{\mathrm{d} F_{N, n . a .}(a)}{\mathrm{d} a} \Delta l-F_{N, n . a .}(a) \frac{\mathrm{d} d_{n . a .}(a)}{\mathrm{d} a}+\frac{\mathrm{d} k_{n . a .}(a)}{\mathrm{d} a} \frac{\Delta l^{2}}{2}+k_{n . a .}(a) \Delta l \frac{\mathrm{d} d_{n . a .}(a)}{\mathrm{d} a}-\pi a^{2} \Delta \gamma \\
& =\left(\frac{\mathrm{d} U_{n . a .}(a)}{\mathrm{d} a}-F_{N, n . a .}(a) \frac{\mathrm{d} d_{n . a .}(a)}{\mathrm{d} a}\right)-\left(\frac{\mathrm{d} F_{N, n . a .}(a)}{\mathrm{d} a} \Delta l-k_{n . a .}(a) \Delta l \frac{\mathrm{d} d_{n . a .}(a)}{\mathrm{d} a}\right)+\frac{\mathrm{d} k_{n . a .}(a)}{\mathrm{d} a} \frac{\Delta l^{2}}{2}-2 \pi a \Delta \gamma \\
& =0
\end{aligned}
$$

It is easy to see that the terms in brackets are identically zero, thus we get

$$
\frac{\mathrm{d} k_{n . a .}(a)}{\mathrm{d} a} \frac{\Delta l^{2}}{2}=2 \pi a \Delta \gamma .
$$

Solution with respect to $\Delta l$ provides Eq. (4). Eqs. (5) and (7) coincide with Eqs. (3) and (2) and provide the solution to the problem. Note that this equation is applicable not only to homogeneous media but to all the media for which the dependence of the contact stiffness on radius is known, in particular of coated, multi-layer or gradient media [3]. In the case of a homogeneous medium $\mathrm{d} k_{\text {n.a. }}(a) / \mathrm{d} a=2 E^{*}$, where $E^{*}$ is the effective elastic modulus responsible for a normal contact problem [4]. In this case we come to the known rule of $\mathrm{He} \beta[5]$.

\section{GenERAl CASE (NOT AXIS-SYMMETRIC OR NON-COMPACT CONTACT AREA)}

If the set of contact configurations of an adhesive contact would repeat that of contact configurations of the normal one for the same shape (which, regrettably, will generally not be the case!), then the adhesive contact could be solved in the following way. For simplicity, we consider here homogeneous media. We assume that the normal contact problem was solved so that the dependence of normal force $F_{N, \text { n.a. }}$ and contact area $A$ on indentation depth $d$ is known: 


$$
\begin{gathered}
F_{N, n . a .}=F_{N, n . a}(d), \\
A_{n . a .}=A_{n . a .}(d) .
\end{gathered}
$$

Now we define the incremental stiffness

$$
k_{n . a .}=\frac{\mathrm{d} F_{N, n . a .}}{\mathrm{d} d}
$$

and the formal "effective contact radius" (which in general case has of course nothing to do with any radius, but is just a formally defined quantity):

$$
a=\frac{k_{n . a .}(d)}{2 E^{*}} .
$$

The normal contact now can be described by the Method of Dimensionality Reduction with the equivalent profile $z=g(x)$, where function $g(x)$ is defined according to

$$
d=g(a),
$$

(just by solving Eq. (15) with respect to $d$, for details see [5]) .

The condition for the equilibrium of an adhesive contact can be obtained from the standard balance of energy at small variation of the "contact radius". We assume that the boundary springs (in the MDR picture) detach when they achieve critical length $\Delta l_{c}$, which is determined by equating the relaxed elastic energy

$$
\Delta U_{e l}=2 \cdot \frac{\mathrm{d} k_{n . a .}}{2 \mathrm{~d} a} \Delta x \Delta l_{c}^{2}
$$

to the change of adhesive energy:

$$
\Delta U_{a d h}=\Delta \gamma \cdot \Delta A=\Delta \gamma \cdot \frac{\mathrm{d} A}{\mathrm{~d} a} \Delta x=\Delta \gamma \cdot \frac{\mathrm{d} A}{\mathrm{~d} d} \frac{\mathrm{d} d}{\mathrm{~d} a} \Delta x=\Delta \gamma \cdot \frac{\mathrm{d} A}{\mathrm{~d} d} \frac{\mathrm{d} g(a)}{\mathrm{d} a} \Delta x .
$$

Equating (17) and (18) we get

$$
2 \cdot \frac{\mathrm{d} k_{\text {n.a. }}}{2 \mathrm{~d} a} \Delta x \Delta l_{c}^{2}=\Delta \gamma \cdot \frac{\mathrm{d} A}{\mathrm{~d} a} \Delta x,
$$

hence

$$
\Delta l_{c}=\sqrt{\frac{2 \Delta \gamma \cdot\left(\mathrm{d} A_{n . a .} / \mathrm{d} a\right)}{\left(\mathrm{d} k_{n . a .} / \mathrm{d} a\right)}} .
$$

In the case of axis-symmetric profiles with a compact contact area we have of course trivially $A=\pi a^{2}, \mathrm{~d} A / \mathrm{d} a=2 \pi a$ and $\Delta l_{c}=\sqrt{2 \pi a \Delta \gamma / E^{*}}$, which coincides with the "rule of Heß" [5]. 


\section{GENERALIZATION FOR ARBITRARY MEDIA}

Note that in the systems with a complicated "microstructure", the surface energy also can depend on the size of the contact, as e.g. illustrated for different shapes with internal damages as well as for "brushes" in [7]. In this case Eq. (22)is modified to

$$
\Delta l_{c}(a)=\sqrt{\frac{2 \Delta \gamma(a) \cdot(\mathrm{d} A(a) / \mathrm{d} a)}{\mathrm{d} k_{\mathrm{na}}(a) / \mathrm{d} a}},
$$

where $\Delta \gamma(a)$ is the size-dependent effective surface energy which can be determined by means of the concept of the "filling factor" the use of which has been validated in many examples in [7]. Together with Eqs. (2) and (3) this solves the problem.

\section{APPLICABILITY OF THE MACROSCOPIC APPROACH TO CONTACT OF ROUGH SURFACES}

Recently, Ciavarella has come independently to a very similar approach [7]. He has also provided an extensive and instructive discussion of applicability of the macroscopic approach to adhesive contacts of rough surfaces. The main assumption of the approach is that the sequence of contact configurations of an adhesive contact is the same as in the case of a non-adhesive contact. This condition is clearly not fulfilled even in the case of "asperity models" like Greenwood and Williamson. There are no reasons to assume that in the case of a more general roughness the contact configurations of an adhesive contact will repeat those of a non-adhesive contact, so that in the general case, this assumption is not fulfilled, either. That the present approach cannot be generally applied to contacts of rough surfaces is already clear from the fact that in the present approach there is no "hysteresis of the force of adhesion" (thus, the force of adhesion does not depend on the loading history which is not the case in real rough contacts as discussed in $[9,10]$. However, there can be some situations where the above assumption is fulfilled or approximately fulfilled. For example, if the Johnson parameter [11] is overcritical then a complete contact can be realized in spite of roughness [12]. Furthermore, the concept can be applied to rough surfaces by using the concept of the filling parameter as discussed in [7]. However, this approach uses the notion of a "real contact area" which is a poorly defined quantity (an excellent discussion of this property and the ways of its proper physical definition can be found in [13]. E.g. one of the "regularizing factors" may be the final range of adhesive forces which substantially modifies the contact situation at a small scale $[14,15]$. Further investigation of this problem, especially using the now available numerical technique of the Boundary Element method for adhesive contacts [7] is needed.

\section{CONCLUSIONS}

Equations (2), (3) and (22) provide a simple solution for all the problems that the normal contact problem has been solved for - either analytically or numerically. This includes all the contacts with a homogeneous continuum, coated medium, gradient 
material, plates, thin layers, membranes or living cells, and so on. The only restriction of the method is that the sequence of the contact configuration is the same as in the nonadhesive problem. This is valid for compact axisymmetric contacts and for some other cases of axisymmetric contacts analyzed in [2].

Acknowledgments: The author acknowledges financial support by the Deutsche Forschungsgemeinschaft (DFG PO 810-55-1). The author acknowledges very useful discussions with M. Ciavarella.

\section{REFERENCES}

1. Argatov, I., Li, Q., Pohrt, R., Popov, V.L., 2016, Johnson-Kendall-Roberts adhesive contact for a toroidal indenter, Proc. R. Soc. A 472, 20160218.

2. Willert, E., Li, Q., Popov, V.L., 2016, The JKR-adhesive normal contact problem of axisymmetric rigid punches with a flat annular shape or concave profiles, Facta Universitatis-Series Mechanical Engineering, 14(3), pp. 281-292.

3. Popov, V.L., Heß, M., Willert, E., 2017, Handbuch der Kontaktmechanik: Exakte Lösungen axialsymmetrischer Kontaktprobleme, Springer, Berlin, 341 p.

4. Popov, V.L., 2017, Contact mechanics and friction. Physical principles and applications. Springer, Berlin.

5. Hess, M., 2011, Über die exakte Abbildung ausgewählter dreidimensionaler Kontakte auf Systeme mit niedrigerer räumlicher Dimension, Cuvillier, Berlin, Germany

6. Popov, V.L., Pohrt, R., Heß, M., General procedure for solution of contact problems under dynamic normal and tangential loading based on the known solution of normal contact problem. The Journal of Strain Analysis for Engineering Design, 51(4), pp. 247-255.

7. Popov, V.L., Pohrt, R., Li, Q., 2017, Strength of adhesive contacts: Influence of contact geometry and material gradients, Friction, 5(3), pp. 308-325.

8. Ciavarella, M. , 2017, An approximate JKR solution for a general contact, including rough contacts, arXiv preprint arXiv:1712.05844

9. Afferrante, L., Ciavarella, M., Demelio, G., 2015, Adhesive contact of the Weierstrass profile, Proc. R. Soc. A, 471, (2182), 20150248.

10. Ciavarella, M., 2017, A note on the possibility of roughness enhancement of adhesion in Persson's theory, International Journal of Mechanical Sciences, 121, pp. 119-122.

11. Johnson, K.L., 1995, The adhesion of two elastic bodies with slightly wavy surfaces, Int. J. Solids Structures, 32(3/4), pp. 423-430.

12. Ciavarella, M., Papangelo, A., 2018, A generalized Johnson parameter for pull-off decay in the adhesion of rough surfaces, Phys Mes., 21(1), pp. 57-75.

13. Ciavarella, M., Papangelo, A., 2017, Discussion of Measuring and Understanding Contact Area at the Nanoscale: A Review (Jacobs, TDB, and Ashlie Martini, A., 2017, ASME Appl. Mech. Rev., 69 (6), p. 060802), Applied Mechanics Reviews, 69(6), 065502. doi: 10.1115/1.4038188

14. Ciavarella, M., Papangelo, A., 2018, A modified form of Pastewka-Robbins criterion for adhesion, The Journal of Adhesion, 94(2), pp. 155-165.

15. Papangelo, A., Ciavarella, M., 2017, A Maugis-Dugdale cohesive solution for adhesion of a surface with a dimple, Journal of The Royal Society Interface, 14(127), 20160996. 